\title{
Pyroelectric Nanogenerators for Harvesting Thermoelectric Energy
}

\author{
Ya Yang, ${ }^{\dagger}$ Wenxi Guo, ${ }^{\dagger}$ Ken C. Pradel, ${ }^{\dagger}$ Guang Zhu, ${ }^{\dagger}$ Yusheng Zhou, ${ }^{\dagger}$ Yan Zhang, ${ }^{\dagger}$ Youfan $\mathrm{Hu}^{\dagger}$ \\ Long Lin, ${ }^{\dagger}$ and Zhong Lin Wang ${ }^{*},+\dagger$ \\ ${ }^{\dagger}$ School of Material Science and Engineering, Georgia Institute of Technology, Atlanta, Georgia 30332-0245, United States \\ ${ }^{\ddagger}$ Beijing Institute of Nanoenergy and Nanosystems, Chinese Academy of Sciences, Beijing, China
}

\section{Supporting Information}

ABSTRACT: Harvesting thermoelectric energy mainly relies on the Seebeck effect that utilizes a temperature difference between two ends of the device for driving the diffusion of charge carriers. However, in an environment that the temperature is spatially uniform without a gradient, the pyroelectric effect has to be the choice, which is based on the spontaneous polarization in certain anisotropic solids due to a time-dependent temperature variation. Using this effect, we experimentally demonstrate the first application of pyroelectric $\mathrm{ZnO}$ nanowire arrays for converting heat energy into electricity. The coupling of the pyroelectric and semiconducting properties in $\mathrm{ZnO}$ creates a polarization electric field and charge separation along the $\mathrm{ZnO}$ nanowire as a result of the time-dependent change in temperature. The fabricated nanogenerator has a good stability, and the characteristic coefficient of heat flow conversion into electricity is estimated to be $\sim 0.05-0.08 \mathrm{Vm}^{2} / \mathrm{W}$. Our study has the potential

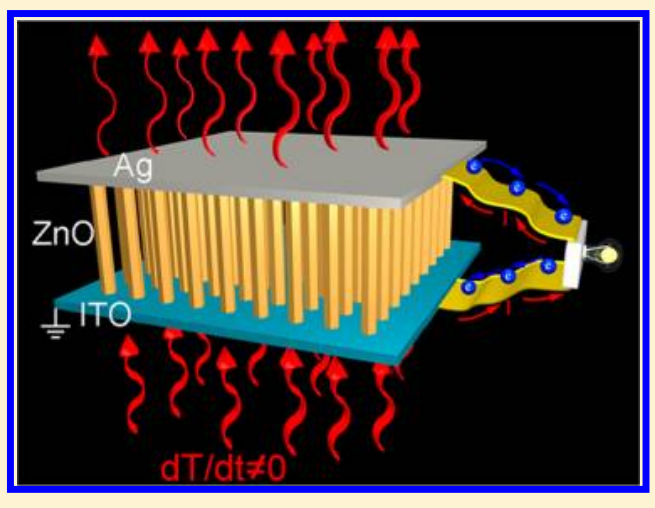
of using pyroelectric nanowires to convert wasted energy into electricity for powering nanodevices.

KEYWORDS: ZnO nanowires, pyroelectric effect, nanogenerators, Schottky contact, Seebeck effect

$\mathrm{W}$ asted heat is a rich source of energy that could be harvested. In 2010, for example, more than $50 \%$ of the energy generated from all sources in the U.S. was lost mainly in the form of wasted heat, ${ }^{1}$ which presents us with a great opportunity to harvest this type of energy using nanotechnology. Harvesting thermoelectric energy mainly relies on the Seebeck effect that utilizes a temperature difference between the two ends of the device for driving the diffusion of charge carriers. ${ }^{2,3}$ The presence of a temperature gradient is a must for the conventional thermoelectric cell. However, in an environment that the temperature is spatially uniform without a gradient, such as in outdoor in our daily life, the Seebeck effect is hardly useful for harvesting thermal energy arising from a time-dependent temperature fluctuation. In this case, the pyroelectric effect is the choice, which is about the spontaneous polarization in certain anisotropic solids as a result of temperature fluctuation, ${ }^{4}$ but there are few studies about using pyroelectric effect for harvesting thermal energy. ${ }^{5}$

Recently, piezoelectric $\mathrm{ZnO}$ nanowires have been effectively used to harvest small-scale mechanical energy. ${ }^{6-9}$ The core of the piezoelectric nanogenerator $(\mathrm{NG})$ is to utilize the piezoelectric potential (piezopotential) in the nanowires created by mechanical straining to drive the flow of electrons in the external load. Piezopotential can be generated by either strain or by temperature due to the anisotropic physical properties of $\mathrm{ZnO}$. Here, we demonstrate the first application of converting heat energy into electricity by means of pyroelectric $\mathrm{ZnO}$ nanowire arrays. By using the coupling of the pyroelectric and semiconducting properties in $\mathrm{ZnO}$, a polarization electric field and charge separation can be created along the $\mathrm{ZnO}$ nanowire as a result of the time-dependent change in temperature. The fabricated nanogenerator shows a good stability, and the characteristic coefficient of heat flow conversion into electricity is about $0.05-0.08 \mathrm{Vm}^{2} / \mathrm{W}$. Our study shows the potential of using pyroelectric nanowires for applications of powering nanodevices, temperature imaging, radiation detection, X-ray generation, and space science. ${ }^{10-12}$

Our design of the pyroelectric NG is to use the anisotropic polarization in $\mathrm{ZnO}$ nanowires created as a result of timedependent temperature fluctuation to drive electron to flow. $\mathrm{ZnO}$ nanowire arrays were grown on ITO substrate via a solution-based growth technique. ${ }^{13}$ Figure $1 \mathrm{a}, \mathrm{b}$ shows that the diameter and length of the nanowires were about $200 \mathrm{~nm}$ and 2 $\mu \mathrm{m}$, respectively. The experimental setup is schematically shown in Figure 1c. An Ag film electrode in contact with the top of the $\mathrm{ZnO}$ nanowires created a Schottky contact, and the ITO electrode at the bottom served as a common electrode for directly connecting the $\mathrm{ZnO}$ nanowires with an external circuit. The $I-V$ curve in Figure 1d shows that the fabricated device exhibited a Schottky behavior at the interface between $\mathrm{Ag}$ and $\mathrm{ZnO}$. To ensure that the electrical signal was coming from the $\mathrm{NG}$, both the forward and reversal connections to the measurement system were introduced for characterizing the output voltage and current. ${ }^{9}$

Received: January 24, 2012

Revised: April 30, 2012 


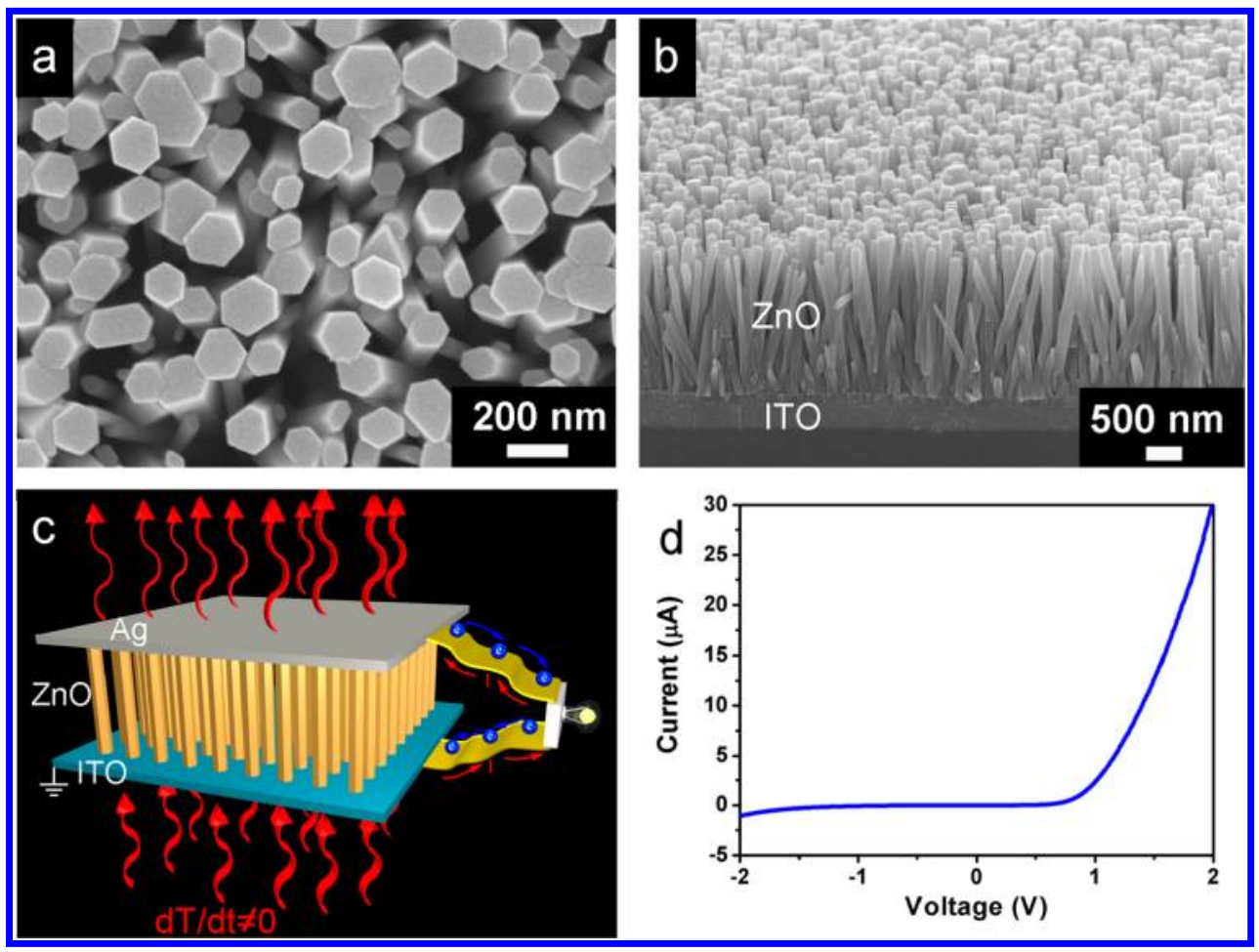

Figure 1. (a) SEM image of the as-grown $\mathrm{ZnO}$ nanowire array. (b) Tilted cross-sectional SEM image of the ZnO nanowire array. (c) Schematic diagram showing the structure of the pyroelectric nanogenerator. (d) $I-V$ characteristics of the nanogenerator measured at room temperature, showing the presence of a Schottky contact between $\mathrm{Ag}$ and $\mathrm{ZnO}$ nanowires.

The open-circuit voltage and short-circuit current of the pyroelectric NG have been measured by varying the temperature in the vicinity of the NG from 295 to $289 \mathrm{~K}$ and from 295 to $304 \mathrm{~K}$ (note the standard room temperature (RT) was 295 $\mathrm{K})$, as shown in Figure 2. Under forward connection, a sharp negative voltage/current pulse $(\sim 5.8 \mathrm{mV} / 108.5 \mathrm{pA})$ was observed when the temperature was quickly decreased from 295 to $289 \mathrm{~K}$ (Figure 2a), and a corresponding positive pulse was received when the temperature was lowed to RT $295 \mathrm{~K}$ (Figure 2b). After reversely connecting the NG to the measurement system, the obtained signals were switched in sign (Figure 2c), suggesting that the measured signals were generated by the fabricated device. When the temperature was rapidly increased from RT 295 to $304 \mathrm{~K}$ (Figure 2d), a positive voltage/current pulse $(\sim 5.8 \mathrm{mV} / 120.4 \mathrm{pA})$ was received (Figure 2e). Again, a reversal connection to the measurement system produced reversed output signal (Figure $2 \mathrm{f}$ ).

To confirm that the obtained signals in Figure 2 were generated by the pyroelectric effect, control experiments were carried out using devices fabricated using different materials. No appreciable jump in voltage/current pulse was observed for an Ag-ITO film or an $\mathrm{Ag}-\mathrm{TiO}_{2}$ nanowire array-ITO structure under the temperature fluctuations (Supporting Information Figures S1 and S2). A linear superposition test (Supporting Information Figure S3) shows that the output current can be enhanced or weakened when the two NGs are connected in parallel with same polarities or opposite polarities, respectively. These results confirm that the observed voltage/current output is indeed generated by the pyroelectric effect of the $\mathrm{ZnO}$ nanowires.

Figure 3 shows the voltage/current output signals of the pyroelectric NG under different magnitude of temperature fluctuations. It can be clearly seen that the voltage/current output peaks were increased with increasing the temperature differences in the device. When the temperature decreased from RT $295 \mathrm{~K}$ to a lower value, a negative voltage/current peak was produced and then returned to zero when there was no temperature change (Figure $3 \mathrm{~b}$ ). The opposite effect was observed when the temperature was recovered back to RT 295 K. However, when the temperature increased from RT $295 \mathrm{~K}$ to a higher value, after a positive voltage/current peak, the current did not return to zero but reached a constant plateau (Figures 2e,f and 3d). The current plateau always existed when the temperature source was maintained (Supporting Information Figure S4). The presence of the plateau is likely due to Seebeck effect in measurement system since a slight temperature drop exists across the NG. It is easy to separate the contribution from Seebeck effect that produces a dc output, while the pyroelectric effect produces an ac output. Details will be presented in the Supporting Information.

Figure $4 \mathrm{a}$ shows the measured voltage/current peaks as a function of temperature change, demonstrating a nearly linear relationship. The pyroelectric current and voltage coefficients can be described as $P_{\mathrm{C}}=[I /(A(\mathrm{~d} T / \mathrm{d} t))]$ and $P_{\mathrm{V}}=[V /(\Delta T$ $\left.r_{\mathrm{d}}\right)$ ], respectively, where $I$ is the pyroelectric current, $V$ is the pyroelectric voltage, $A$ is the electrode area, $\mathrm{d} T / \mathrm{d} t$ is the rate of change in temperature, $\Delta T$ is the change of temperature, and $r_{\mathrm{d}}$ is the Debye length of $\mathrm{ZnO}$ (about $30 \mathrm{~nm}$ ). ${ }^{14,15}$ Using our experimental data, we estimated pyroelectric current and voltage coefficients of $\sim 1.2-1.5 \mathrm{nC} / \mathrm{cm}^{2} \mathrm{~K}$ and $\sim 2.5-4.0 \times$ $10^{4} \mathrm{~V} / \mathrm{mK}$, respectively (Figure $4 \mathrm{~b}$ ); the former is larger than those reported for $\mathrm{ZnO}$ bulk $\left(0.94 \mathrm{nC} / \mathrm{cm}^{2} \mathrm{~K}\right)$ and film $(1.0$ $\left.\mathrm{nC} / \mathrm{cm}^{2} \mathrm{~K}\right)$ materials. ${ }^{10,16}$ As compared with bulk/film-structured $\mathrm{ZnO}$, the enhancement of the pyroelectric coefficients in $\mathrm{ZnO}$ nanowire arrays is likely due to the single crystalline 


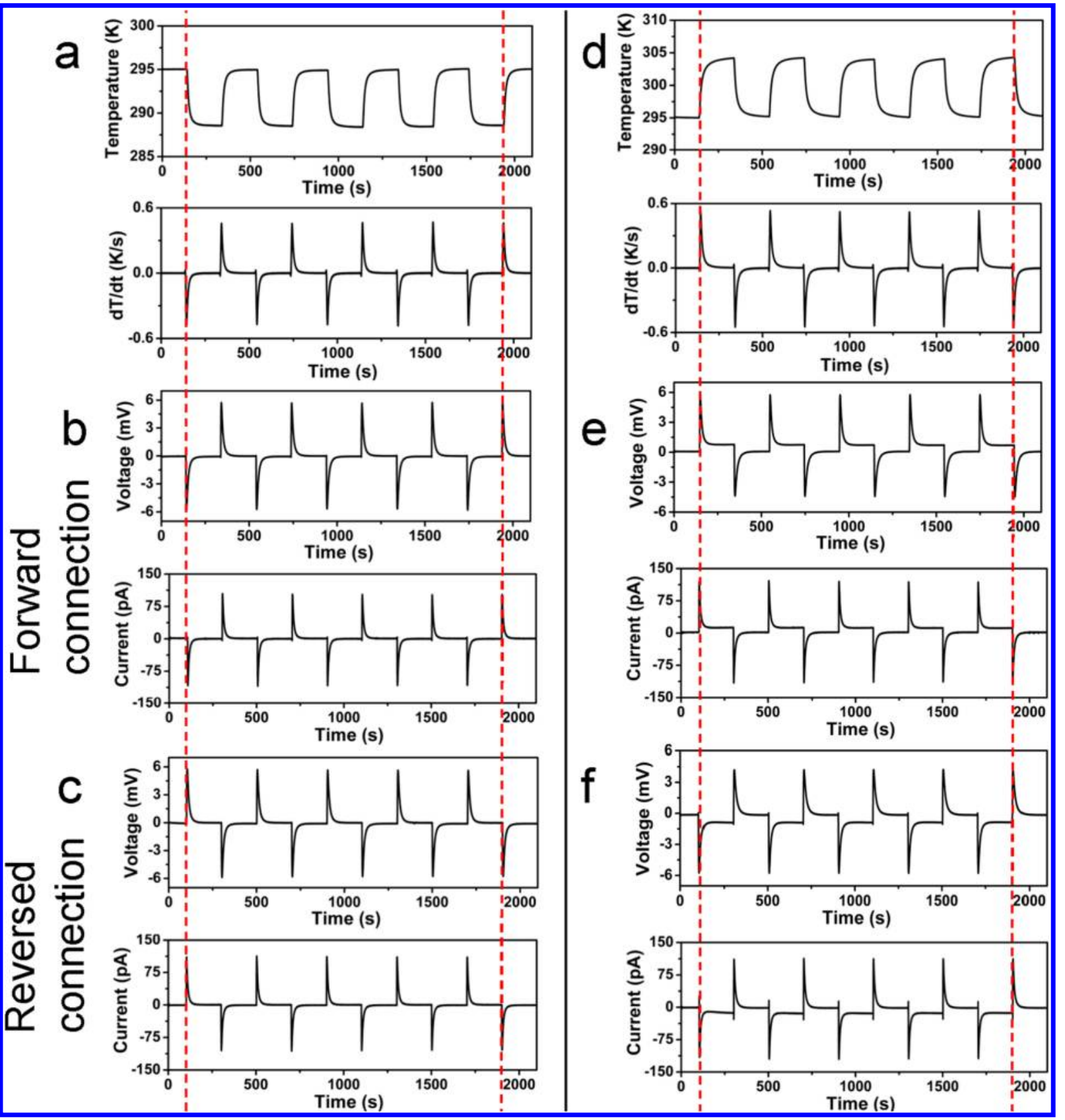

Figure 2. $(\mathrm{a}, \mathrm{d})$ The cyclic changes in temperature of the nanogenerator and the corresponding differential curve. (b,c,e,f) Measured open-circuit voltage and short-circuit current of the pyroelectric nanogenerator at forward connection and reversed connection to the measurement system, when it was subject to a repeated temperature change from (b,c) RT 295 to $289 \mathrm{~K}$ (below RT) and (e,f) RT 295 to $304 \mathrm{~K}$ (above RT), respectively.

structures with the same preferred orientations along the $c$-axis and possibly less dislocation density.

The parameter $P_{\mathrm{f}}=\mathrm{d} F / \mathrm{d} Q \approx V\left(t_{0}\right) /\left[Q\left(t_{0}\right) r_{\mathrm{d}}\right]$ was used to characterize the ability of the NG to convert the thermal flow into electricity, where $F$ is the electric field created by the pyroelectricity and $Q$ is a thermal flow per unit volume. ${ }^{15}$ Here the value of $Q\left(t_{0}\right)$ is given by $Q\left(t_{0}\right)=\left[(S h) / V_{0}\right]\left(T_{0}-T_{\infty}\right)$, where $h$ is the heat transfer coefficient, and $V_{0}$ and $S$ are the sample volume and surface area, respectively. The obtained characteristic coefficient $P_{\mathrm{f}}$ of heat flow conversion into electricity is $\sim 0.05-0.08 \mathrm{Vm}^{2} / \mathrm{W}$ (Figure $4 \mathrm{c}$ ), which is 10 times larger than that of the reported for GaN film $(\sim 2.3 \times$ $\left.10^{-3} \mathrm{Vm}^{2} / \mathrm{W}\right) .^{15}$ The stability of the NG was examined by a continuous and constant change of temperature for 200 cycles within $6 \mathrm{~h}$ (Figure 4d). The output voltage signal was very stable with no declining trend.

Usually, the pyroelectric coefficient is defined as the differential change of spontaneous polarization due to a change in temperature. When the dimensions and volume of the material are fixed, the primary pyroelectric coefficient can be used to describe the charges produced under a change in temperature. If the material's dimensions/volume can change due to a change in temperature, strain will be induced due to the anisotropic deformation of the material, resulting in an additional contribution of piezoelectrically induced charges. It is commonly described as a secondary pyroelectric effect, which is likely more important for wurtzite crystals (such as $\mathrm{ZnO}$, $\mathrm{CdS}) .{ }^{16,17}$ By using COMSOL software, we calculated the 


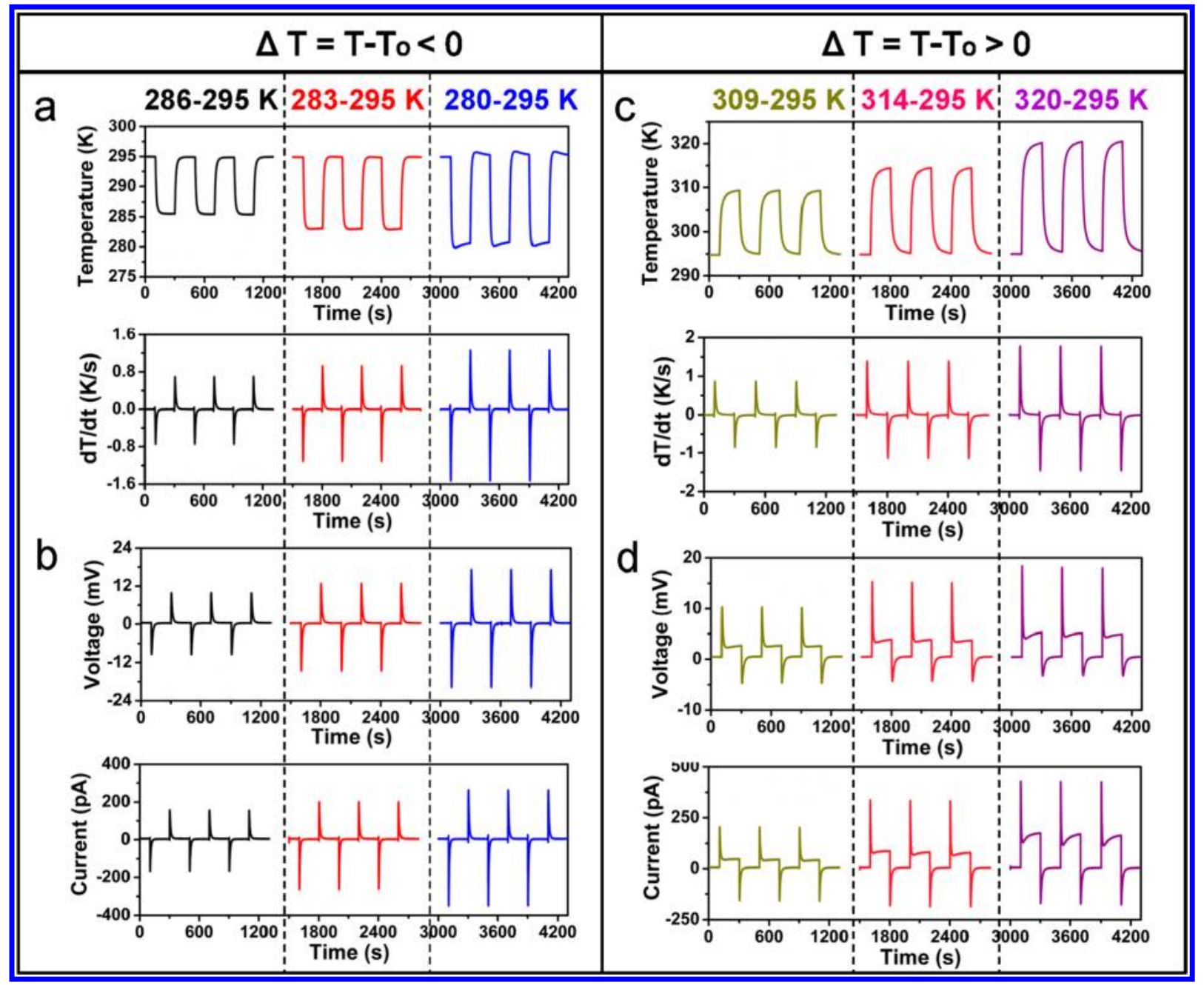

Figure 3. Dropping of the temperature from RT $\left(T_{0}=295 \mathrm{~K}\right)$ (a) and the corresponding output voltage and current (b). Raising temperature from RT $\left(T_{0}=295 \mathrm{~K}\right)(\mathrm{c})$ and the corresponding output voltage and current $(\mathrm{d})$. The observed plateaus in output voltage and current in the case of raising temperature in (d) is likely due to Seebeck effect and will be discussed in Supporting Information.

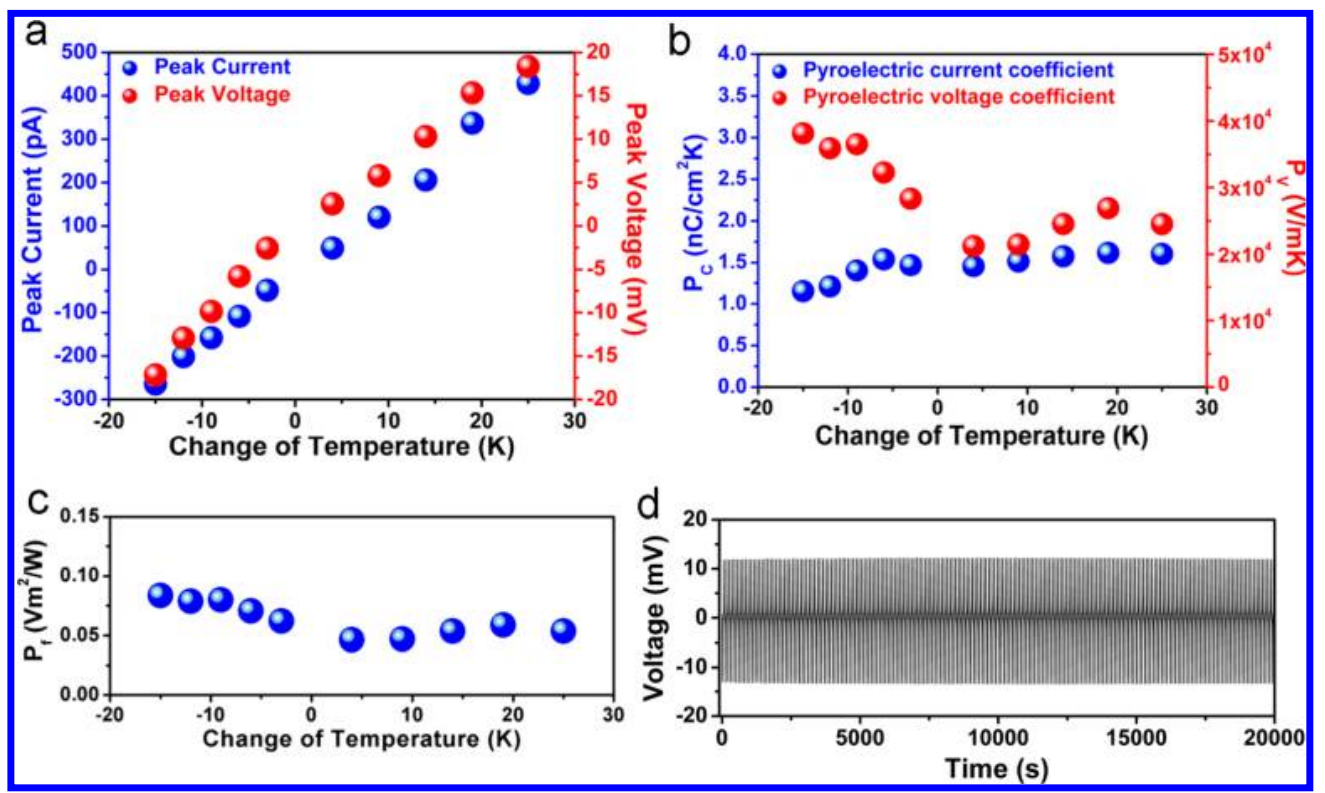

Figure 4. The output peak voltage/current (a), pyroelectric voltage/current coefficients (b), and energy conversion characteristic value (c), as a function of change in temperature. (d) Output stability test of the nanogenerator through cyclic temperature change for over 200 cycles. 


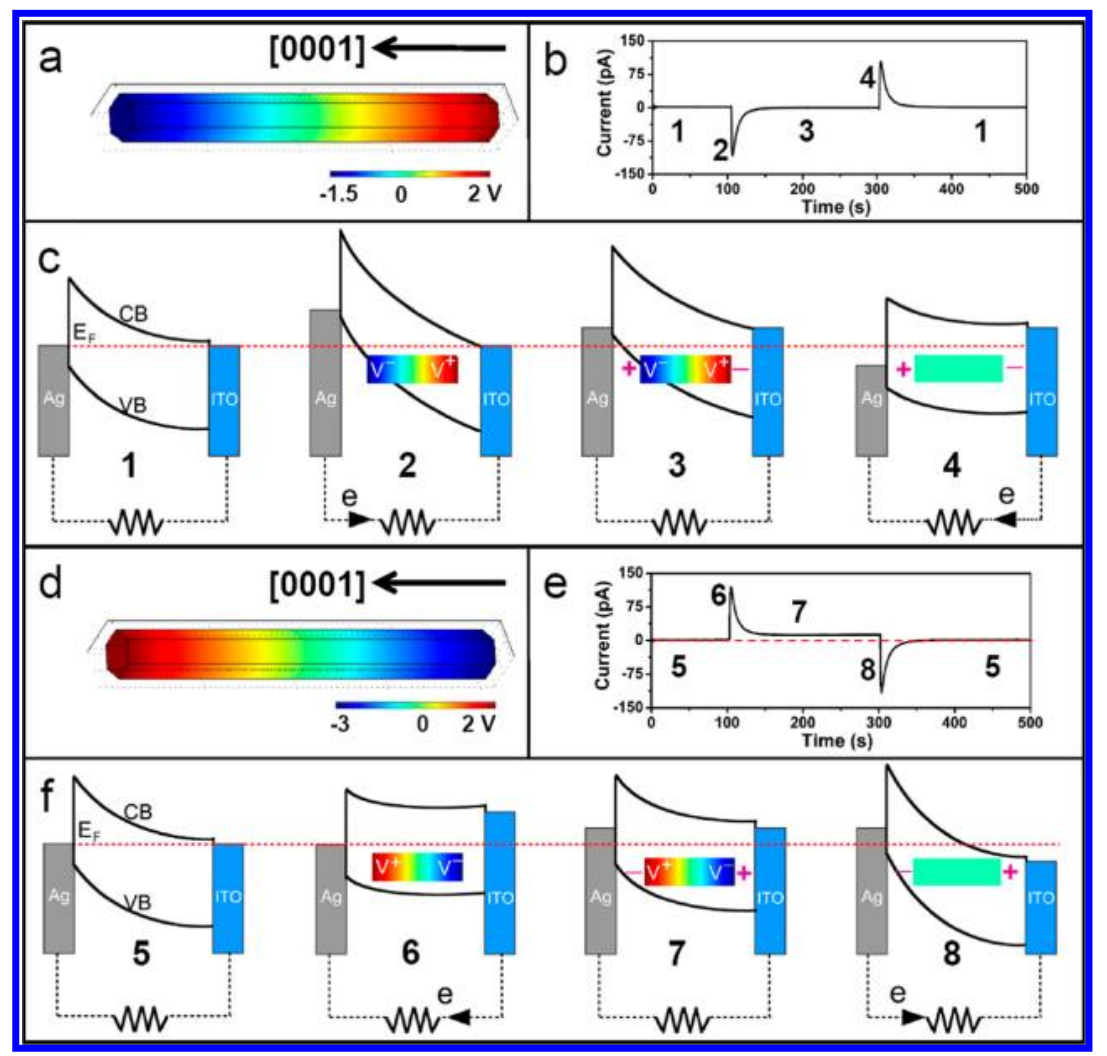

Figure 5. (a) The calculated electrical potential distribution in a single $\mathrm{ZnO}$ nanowire when the temperature is decreased from RT 295 to $289 \mathrm{~K}$. $(b, c)$ Experimentally measured output current and the corresponding energy band diagrams of the device at the four different stages (" $1-4$ "). (d) The calculated electrical potential distribution of a single $\mathrm{ZnO}$ nanowire along the [0001] direction when the temperature increased from RT 295 to $304 \mathrm{~K} .(\mathrm{e}, \mathrm{f})$ Experimentally measured output current and the corresponding energy band diagrams of the nanogenerator at the four different stages (“5-8”).

electrical potential distribution in a single $\mathrm{ZnO}$ nanowire due to the change in temperature, as shown in Figure 5. For simplicity of the calculation, the doping in $\mathrm{ZnO}$ is ignored, so that it is treated as an insulator. In the calculation, the diameter and length of the $\mathrm{ZnO}$ nanowire are $200 \mathrm{~nm}$ and $2 \mu \mathrm{m}$, respectively. The results show that an electrical potential distribution from 2.1 to $-1.7 \mathrm{~V}$ (Figure 5a) was created along [0001] direction when the temperature was decreased from RT 295 to $289 \mathrm{~K}$. An electrical potential distribution from -3.1 to $2.6 \mathrm{~V}$ (Figure $5 \mathrm{~d})$ was obtained by raising the temperature from RT 295 to $304 \mathrm{~K}$. Although the calculated electrical potential is up to $3 \mathrm{~V}$, the actual electrical potential in semiconducting $\mathrm{ZnO}$ nanowires is much lower due to the screening effect of the free charge carriers. ${ }^{18,19}$ Thus we observed the much lower output voltage peaks in Figures 2 and 3.

Figure 1d clearly demonstrates that the Schottky barriers appear at the contacts, where Ag and ITO are the positive and negative electrodes, respectively. The device structure of $\mathrm{Ag} /$ $\mathrm{ZnO} / \mathrm{ITO}$ can be considered as a $\mathrm{ZnO}$ layer sandwiched between two back-to-back Schottky barriers at the contacts. Under a positive bias, the electrons will flow along ITO/ZnO/ $\mathrm{Ag}$, where the reversed Schottky barrier between ITO and $\mathrm{ZnO}$ is dominant. As compared with the current values under the negative biases in Figure 1d, the much larger current values under the positive biases indicate that the Schottky barrier height between ITO and $\mathrm{ZnO}$ is much lower than that between $\mathrm{Ag}$ and $\mathrm{ZnO}$, as shown in Figure 5. Figures 5c,f show the proposed mechanisms for the generation of current in terms of the band structure of the system under temperature changes from RT 295 to $289 \mathrm{~K}$ (Figure 5c) and RT 295 to $304 \mathrm{~K}$
(Figure 5f), respectively. The regions marked with " $1-8$ " in Figures $5 b$,e correspond to the numbered diagrams in Figures $5 c, f$, respectively.

When the $\mathrm{ZnO}$ nanowire is subjected to a drop in temperature from RT 295 to $289 \mathrm{~K}$, a negative pyroelectric field (Figure 5a) is created in the $\mathrm{ZnO}$ nanowire along the [0001] direction. The negative and positive pyroelectric potentials $\left(V^{-}\right.$and $\left.V^{+}\right)$sides are in contact with the top $\mathrm{Ag}$ electrode and the bottom ITO electrode, respectively. The negative electrical potential $\left(V^{-}\right)$can lift up the local conduction band of $\mathrm{ZnO}$ and Fermi level of the $\mathrm{Ag}$ electrode by $\Delta E=e\left(V^{+}-V^{-}\right)$, which can drive electrons to flow from $\mathrm{Ag}$ electrode to ITO electrode through an external load resistor. This is consistent with the observed negative current (" 2 " in Figure $5 b$ ). When a new equilibrium value was then created, there will be no observed current output (" 3 " in Figure 5c). When the temperature returned to RT $295 \mathrm{~K}$, the pyroelectric potential disappears. The accumulated free charges at the ITO electrode will flow back to the Ag electrode ("4" in Figure 5c). When the temperature was increased from RT 295 to $304 \mathrm{~K}$, the electrical potential will become negative at the ITO electrode and positive at the Ag electrode (Figure 5d), respectively. The conduction band and Fermi level of the ITO electrode are raised by $\Delta E$ due to the negative electrical potential $\left(V^{-}\right)$, driving the electrons to flow from the ITO electrode to the Ag electrode ("6" in Figure 5f). There should be no observed current output after a new equilibrium value appears. It is noticed that the current plateau (" 7 " in Figure 5f) is likely due to Seebeck effect not pyroelectric effect (see the Supporting Information). After the temperature returned to RT 
$295 \mathrm{~K}$, the accumulated electrons at the Ag electrode are released due to the disappearance of electrical potential and thus flow back to the ITO electrode (" 8 " in Figure 5f).

In summary, we have experimentally demonstrated the first pyroelectric nanogenerator based on $\mathrm{ZnO}$ nanowire arrays for harvesting thermoelectric energy. The pyroelectric current and voltage coefficients for the $\mathrm{ZnO}$ nanowires with the average diameters of $200 \mathrm{~nm}$ are $\sim 1.2-1.5 \mathrm{nC} / \mathrm{cm}^{2} \mathrm{~K}$ and $\sim 2.5-4.0 \times$ $10^{4} \mathrm{~V} / \mathrm{mK}$, respectively. The energy conversion characteristic coefficient of the NG is $\sim 0.05-0.08 \mathrm{Vm}^{2} / \mathrm{W}$. This new type of NG can be the basis for self-powered nanotechnology that harvests thermal energy from the time-dependent temperature fluctuation in our environment for applications such as wireless sensors, temperature imaging, medical diagnostics, and personal microelectronics.

Methods Summary. Growth of ZnO Nanowire Arrays. A $50 \mathrm{~nm}$ thick $\mathrm{ZnO}$ seed layer was deposited on a $500 \mathrm{~nm}$ thick ITO layer on a thin glass substrate. $\mathrm{ZnO}$ nanowires were grown on the $\mathrm{ZnO}$ seed layer using a hydrothermal method. An aqueous solution of hexamethylenetetramine and $\mathrm{Zn}$ $\left(\mathrm{NO}_{3}\right)_{2} \cdot 6 \mathrm{H}_{2} \mathrm{O}$ in equal concentrations $(50 \mathrm{mM})$ was used in the growth of $\mathrm{ZnO}$ nanowire arrays. Because of surface tension, the substrate floated on the solution surface with one face (ITO) down. Growth of $\mathrm{ZnO}$ nanowire arrays was carried out in a mechanical convection oven at $85{ }^{\circ} \mathrm{C}$ for $10 \mathrm{~h}$.

Fabrication and Measurement of the Nanogenerator. A silver film was deposited on the top of $\mathrm{ZnO}$ nanowire array and served as the top electrode of the nanogenerator. The area of the nanogenerator was $\sim 15 \mathrm{~mm}^{2}$. The thermoelectric-based heater and cooler were used to change the temperature of the device. A temperature sensor was used to record the temperature of $\mathrm{ZnO}$ nanowire array during all experiments. The output performance of the nanogenerator was measured using a low-noise voltage/current preamplifier without introducing any external power source to circuit.

\section{ASSOCIATED CONTENT}

\section{S Supporting Information}

Additional figures about the control experiments, the linear superposition of two pyroelectric nanogenerators in parallel, output current of pyroelectric nanogenerator under a change in temperature for a long time (about $1 \mathrm{~h}$ ), and the mechanism of the observed dc current plateau under a change in temperature in Figure 3d. This material is available free of charge via the Internet at http://pubs.acs.org.

\section{AUTHOR INFORMATION}

\section{Corresponding Author}

*E-mail: zlwang@gatech.edu.

\section{Notes}

The authors declare no competing financial interest.

\section{ACKNOWLEDGMENTS}

This work was supported by BES DOE (DE-FG0207ER46394).

\section{REFERENCES}

(1) Lawrence Livermore National Laboratory, "Estimated energy use in 2010: 98 quads". https://flowcharts.llnl.gov/, (accessed 2010).

(2) DiSalvo, F. J. Science 1999, 285, 703-706.

(3) Bell, L. E. Science 2008, 321, 1457-1461.

(4) Lang, S. B. Sourcebook of pyroelectricity; Gordon \& Breach Science: London, 1974.
(5) Morozovska, A. N.; Eliseev, E. A.; Svechnikov, G. S.; Kalinin, S. V. J. Appl. Phys. 2010, 108, 042009.

(6) Wang, Z. L.; Song, J. H. Science 2006, 14, 242-246.

(7) Wang, X. D.; Song, J. H.; Liu, J.; Wang, Z. L. Science 2007, 316, $102-105$.

(8) Qin, Y.; Wang, X. D.; Wang, Z. L. Nature 2008, 451, 809-813.

(9) Yang, R. S.; Qin, Y.; Dai, L. M.; Wang, Z. L. Nat. Nanotechnol. 2009, 4, 34-39.

(10) Lang, S. B. Phys. Today 2005, 58, 31-36.

(11) Cooper, J. Nature 1962, 194, 269-271.

(12) Brownridge, J. D. Nature 1992, 358, 287-288.

(13) Hu, Y. F.; Zhang, Y.; Xu, C.; Lin, L.; Snyder, R. L.; Wang, Z. L. Nano Lett. 2011, 11, 2572-2577.

(14) Lang, S. B.; Tofail, S. A. M.; Gandhi, A. A.; Gregor, M.; WolfBrandstetter, C.; Kost, J.; Bauer, S.; Krause, M. Appl. Phys. Lett. 2011, 98, 123703.

(15) Bykhovski, A. D.; Kaminski, V. V.; Shur, M. S.; Chen, Q. C.; Khan, M. A. Appl. Phys. Lett. 1996, 69, 3254-3256.

(16) Ye, C.; Tamagawa, T.; Polla, D. L. J. Appl. Phys. 1991, 70, 55385543.

(17) Zook, J. D.; Liu, S. T. J. Appl. Phys. 1978, 49, 4604-4606.

(18) Gao, Y.; Wang, Z. L. Nano Lett. 2009, 9, 1103-1110.

(19) Yang, Y.; Guo, W.; Zhang, Y.; Ding, Y.; Wang, X.; Wang, Z. L. Nano Lett. 2011, 11, 4812-4817. 